\title{
INVASIVE PULMONARY ASPERGILLOSIS AND LUPUS NEPHRITIS FLARE: A CASE REPORT
}

Pedro Serra de Candol ${ }^{1, \star}$, Melissa Adriana Torres González ${ }^{1}$, Taíssa Cerqueira dos Santos ${ }^{1}$, Thaynah Pisani Lisboa ${ }^{1}$, Eduardo Florim Terra ${ }^{1}$, Camila Souto Oliveira Elias ${ }^{1}$, Camila Pitasi Arguelhes ${ }^{1}$

1.Universidade do Estado do Rio de Janeiro, Rio de Janeiro (RJ), Brazil.

*Corresponding author: pedrocandol@hotmail.com

\section{BACKGROUND}

Invasive aspergillosis is a life-threatening fungal infection with predominantly pulmonary involvement, especially in immunosuppressed patients with prolonged neutropenia. This infection rarely occurs in systemic lupus erythematosus (SLE) and risk factors in this population were active disease, neutropenia and immunosuppressive therapy.

\section{CASE REPORT}

A 29-year-old woman with SLE and proliferative nephritis in sustained remission for 9 years after cyclophosphamide induction treatment ( $5.9 \mathrm{~g}$ cumulative dose), currently using low-dose hydroxychloroquine and azathioprine $(0.7 \mathrm{mg} / \mathrm{kg} / \mathrm{day})$, was hospitalized with weight loss, asthenia, vespertine fever, night sweats, dyspnea and secretive cough. In social history, there was a report of daily contact with sand, soil and fertilizer for planting. Laboratory tests showed mild anemia, leukopenia with severe lymphopenia, increase of inflammatory markers, transaminases and canalicular enzymes, in addition to nephrotic proteinuria, urinary erythrocyte dysmorphism, anti-DNA in high titers and consumed complement.

Chest tomography evidencing extensive bilateral involvement with focal points of consolidation and ground-glass opacity attenuation, mostly with signs of inverted halo. Radiological aspect suggesting fungal infection, tuberculosis or cryptogenic organizing pneumonia associated with lupus activity (Fig. 1).

In the microbiological investigation, the following tests yielded negative results: two sputum smears, tuberculin skin test, molecular test for diagnosis of coronavirus disease 2019 (COVID-19), histoplasma antibody immunodiffusion test, galactomannan and cryptococcal antigen latex agglutination test. She was submitted to bronchoscopy with bronchoalveolar lavage showing negative results for culture of common germs, bacterioscopy, bacilloscopy and GeneXpert molecular test for Mycobacterium tuberculosis. Direct mycological examination evidenced many typical septate, branched and irregular hyphae and fungal culture revealed growth of Aspergillus sp., confirming invasive pulmonary Aspergillosis diagnosis. Therapy was introduced with voriconazole $4 \mathrm{mg} / \mathrm{kg} / \mathrm{day}$, with excellent clinical and radiological response, enabling to subsequent nephritis therapy after 4 weeks of antifungal treatment.

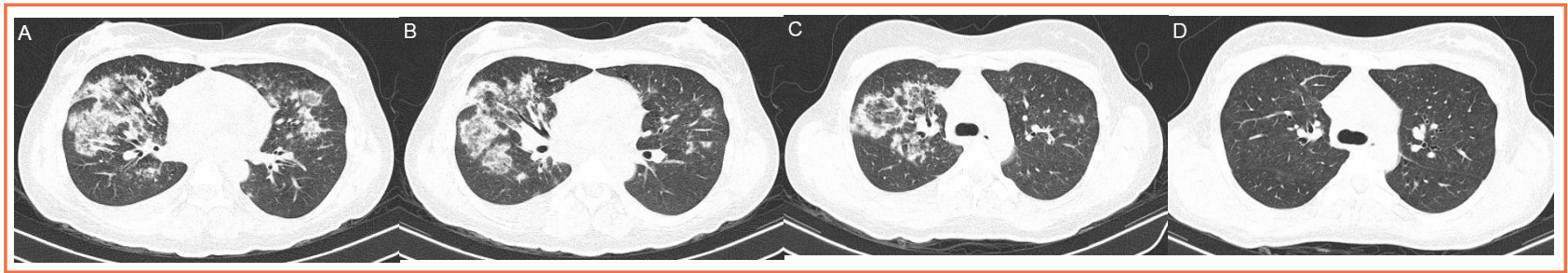

Figure 1. (A-C) Radiological findings of consolidation areas with central ground-glass opacity attenuation, configuring the reversed halo sign. (D) Control chest tomography after 2 weeks of treatment with voriconazole, showing resolution of consolidations and ground-glass opacities.

\section{CONCLUSION}

This case report emphasizes a high significance of suspicion for indolent infections, even in the most unusual case scenarios, which may simulate disease activity or even trigger it. In this case, the patient made use of low-dose of immunosuppressants and did not display any other risk factors as portrayed in medical literature for invasive pulmonary aspergillosis, infection with high morbidity and mortality, if not treated early. 